\title{
Applications of Psychological Science for Actionable Analytics
}

\author{
Di Chen, Wei Fu, Rahul Krishna, Tim Menzies \\ North Carolina State University, USA \\ Raleigh, NC \\ \{dchen20,wfu,rkrish11\}@ncsu.edu,tim.menzies@gmail.com
}

\begin{abstract}
Actionable analytics are those that humans can understand, and operationalize. What kind of data mining models generate such actionable analytics? According to psychological scientists, humans understand models that most match their own internal models, which they characterize as lists of "heuristic" (i.e., lists of very succinct rules). One such heuristic rule generator is the Fast-andFrugal Trees (FFT) preferred by psychological scientists. Despite their successful use in many applied domains, FFTs have not been applied in software analytics. Accordingly, this paper assesses FFTs for software analytics.

We find that FFTs are remarkably effective. Their models are very succinct ( 5 lines or less describing a binary decision tree). These succinct models outperform state-of-the-art defect prediction algorithms defined by Ghortra et al. at ICSE'15. Also, when we restrict training data to operational attributes (i.e., those attributes that are frequently changed by developers), FFTs perform much better than standard learners.

Our conclusions are two-fold. Firstly, there is much that software analytics community could learn from psychological science. Secondly, proponents of complex methods should always baseline those methods against simpler alternatives. For example, FFTs could be used as a standard baseline learner against which other software analytics tools are compared.
\end{abstract}

\section{KEYWORDS}

Decision trees, heuristics, software analytics, psychological science, empirical studies, defect prediction

\section{ACM Reference Format:}

Di Chen, Wei Fu, Rahul Krishna, Tim Menzies. 2018. Applications of Psychological Science for Actionable Analytics. In Proceedings of Florida (FSE'18). ACM, New York, NY, USA, 12 pages. https://doi.org/XX.YY/ZZ

\section{INTRODUCTION}

Data mining tools have been applied to many applications in Software Engineering (SE). For example, it has been used to estimate how long it would take to integrate new code into an existing project [15], where defects are most likely to occur [46, 55], or how long will it take to develop a project $[33,66]$, etc. Large organizations like Microsoft routinely practice data-driven policy

Permission to make digital or hard copies of part or all of this work for personal or classroom use is granted without fee provided that copies are not made or distributed for profit or commercial advantage and that copies bear this notice and the full citation on the first page. Copyrights for third-party components of this work must be honored.

For all other uses, contact the owner/author(s)

FSE'18, Nov 2018

(C) 2018 Copyright held by the owner/author(s)

ACM ISBN ZZ-YY-24-ZZ/QQ/A...\$15.00

https://doi.org/XX.YY/ZZ development where organizational policies are learned from an extensive analysis of large datasets [6, 65].

Despite these successes, there exists some drawbacks with current software analytic tools. At a recent workshop on "Actionable Analytics" at ASE'15, business users were very vocal in their complaints about analytics [27], saying that there are rarely producible models that business users can understand or operationalize.

Accordingly, this paper explores methods for generating actionable analytics for:

- Software defect prediction;

- Predicting close time for Github issues.

There are many ways to define "actionable" but at the very least, we say that something is actionable if people can read and use the models it generates. Hence, for this paper, we assume:

\section{Actionable $=$ Comprehensible + Operational .}

We show here that many algorithms used in software analytics generate models that are not actionable. Further, a data mining algorithm taken from psychological science [9, 14, 22-24, 42, 43, 54], called Fast-and-Frugal trees $\left(\mathrm{FFTs}^{1}\right)$, are very actionable.

Note that demanding that analytics be actionable also imposes certain restrictions on (a) the kinds of models that can be generated and (b) the data used to build the models.

(a) Drawing on psychological science, we say an automatically generated model is comprehensible if:

- The model matches the models used internally by humans; i.e., it comprises small rules.

- Further, for expert-level comprehension, the rules should quickly lead to decisions (thus freeing up memory for other tasks).

For more on this point, see Section 2.2.

(b) As to operational, we show in the historical log of software projects that only a few of the measurable project attributes are often changed by developers. For a data mining algorithm to be operational, it must generate effective models even if restricted to using just those changed attributes.

Using three research questions, this paper tests if these restrictions damage our ability to build useful models.

RQ1: Do FFTs models perform worse than the current stateof-the-art? We will find that:

\footnotetext{
${ }^{1}$ The reader might be aware that FFT is also an acronym for "Fast Fourier Transform". Apparently, the psychological science community was unaware of that acronym when they named this algorithm.
} 
For defect prediction, FFTs out-perform the state-of-art. When compared to state-of-the-art defect prediction algorithms surveyed by Ghotra et al. [21], FFTs are more effective (where "effective" is measured in terms of a recall/false alarm metric or the $P_{o p t}$ metric defined in $\S 3.4$ ).

RQ2: Are FFTs more operational than the current state-ofthe-art? This research question tests what happens when we learn from less data; i.e., if we demand our models avoid using attributes that are rarely changed by developers. We show that:

When learning from less data, FFTs performance is stabler than some other learners.

When data is restricted to attributes that developers often change, then FFTs performance is only slightly changed while the performance of some other learners, can vary by alarmingly large amounts.

The observed superior performance of FFT raises the question:

RQ3: Why do FFTs work so well? Our answer to this question will be somewhat technical but, in summary we will say:

FFTs match the structure of SE data

SE data divides into a few regions with very different properties and FFTs are good way to explore such data spaces.

In summary, the contributions of this paper are:

- A novel inter-disciplinary contribution of the application of psychological science to software analytics.

- A cautionary tale that, for software analytics, more complex learners can perform worse.

- A warning that many current results in software analytics make the, possibly unwarranted, assumption that merely because an attribute is observable, that we should use those attributes in a model.

- Three tests for "actionable analytics": (a) Does a data mining produce succinct models? (b) Do those succinct models perform as well, or better, than more complex methods? (c) If the data mining algorithm is restricted to just the few attributes that developers actually change, does the resulting model perform satisfactorily?

- A demonstration that the restraints demanding by actionable analytics (very simple models, access to less data) need not result in models with poor performance.

- A new, very simple baseline data mining method (FFTs) against which more complex methods can be compared.

- A reproduction package containing all the data and algorithms of this paper, see http://url_blinded_for_review.

The rest of this paper is structured as follows. In Section 2, we introduce the concepts of "operational" and "comprehensible" as the preliminaries. Our data, experimentation settings and evaluation measures will be described in Section 3. In Section 4, we show our results and answer to research questions. Threats and validity of our work is given in Section 5. In Section 6, we conclude this paper with the following:

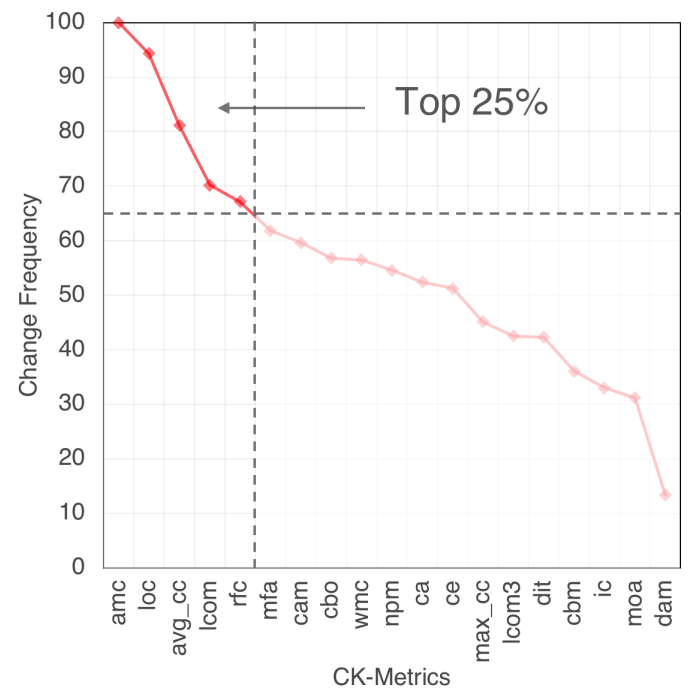

Figure 1: Only some metrics change between versions $i$ and $i+1$ of a software system. For definitions of the metrics on the $x$-axis, see Table 1 . To create this plot, we studied the 26 versions of the ten datasets in Table 3. First we initialize total $=0$, then for all pairs of versions $i, i+1$ from the same data set, we (a) incremented total by one; (b) collected the distributions of metric $m$ seen in version $i$ and $i+1$ of the software; (c) checked if those two distributions were different; and if so, (d) added one to changed $_{m}$. Afterwards, the y-axis of this plot was computed using $100 *$ changed $_{m} /$ total. $_{\text {. }}$

- There is much the software analytics community could learn from psychological science.

- Proponents of complex methods should always baseline those methods against simpler alternatives.

Finally, we discuss future work.

\section{PRELIMINARIES}

\subsection{Operational}

This paper assumes that for a data mining algorithm to be operational, it must generate effective models even if restricted to using just those attributes which, in practice, developers actually change. We have two reasons for making that assumption.

Firstly, this definition of operational can make a model much more acceptable to developers. If a model says that, say, $x>0.6$ leads to defective code then developers will ask for guidance on how to reduce " $x$ " (in order to reduce the chances of defects). If we define "operational" as per this article, then it is very simple matter to offer that developer numerous examples, from their own project's historical log, of how " $x$ " was changed.

Secondly, as shown in Figure 1 there exist attributes that are usually not changed from one version to the next. Figure 1 is important since, as shown in our RQ2 results, when we restrict model construction to just the $25 \%$ most frequently changed attributes, this can dramatically change the behavior of some data mining algorithms (but not FFTs). 
Technical aside: in Figure 1, we defined "changed" using the A12 test [67] which declares two distributions different if they differ by more than a small effect. A recent ICSE'11 article [5] endorsed the use of A12 due to its non-parametric nature, it avoids any possibly incorrect Gaussian assumptions about the data.

\subsection{Comprehensible}

Why Demand Comprehensibility? This paper assumes that better data mining algorithms are better at explaining their models to humans. But is that always the case?

The obvious counter-argument is that if no human ever needs to understand our audited model, then it does not need to be comprehensible. For example, a neural net could control the carburetor of an internal combustion engine since that carburetor will never dispute the model or ask for clarification of any of its reasoning.

On the other hand, if a model is to be used to persuade software engineers to change what they are doing, it needs to be comprehensible so humans can debate the merits of its conclusions. Several researchers demand that software analytics models needs to be expressed in a simple way that is easy for software practitioners to interpret [16, 39, 45]. According to Kim et al. [32], software analytics aim to obtain actionable insights from software artifacts that help practitioners accomplish tasks related to software development, systems, and users. Other researchers [64] argue that for software vendors, managers, developers and users, such comprehensible insights are the core deliverable of software analytics. Sawyer et al. comments that actionable insight is the key driver for businesses to invest in data analytics initiatives [62]. Accordingly, much research focuses on the generation of simple models, or make blackbox models more explainable, so that human engineers can understand and appropriately trust the decisions made by software analytics models $[1,19]$.

If a model is not comprehensible, there are some explanation algorithms that might mitigate that problem. For example:

- In secondary learning, the examples given to a neural network are used to train a rule-based learner and those learners could be said to "explain" the neural net [13].

- In contrast set learning for instance-based reasoning, data is clustered and users are shown the difference between a few exemplars selected from each cluster [35].

Such explanation facilities are post-processors to the original learning method. An alternative simpler approach would be to use learners that generate comprehensible models in the first place.

The next section of this paper discusses one such alternate approach for creating simple comprehensible models.

Theories of Expert Comprehension. Psychological science argues that models comprising small rules are more comprehensible. This section outlines that argument.

Larkin et al. [36] characterize human expertise in terms of very small short term memory, or STM (used as a temporary scratch pad for current observation) and a very large long term memory, or LTM. The LTM holds separate tiny rule fragments that explore the contents of STM to say "when you see THIS, do THAT". When an LTM rule triggers, its consequence can rewrite STM contents which, in turn, can trigger other rules.
Short term memory is very small, perhaps even as small as four to seven items $[12,52]^{2}$. Experts are experts, says Larkin et al. [36] because the patterns in their LTM patterns dictate what to do, without needing to pause for reflection. Novices perform worse than experts, says Larkin et al., when they fill up their STM with too many to-do's where they plan to pause and reflect on what to do next. Since, experts post far fewer to-do's in their STMs, they complete their tasks faster because (a) they are less encumbered by excessive reflection and (b) there is more space in their STM to reason about new information. While first proposed in 1981, this STM/LTM theory still remains relevant [40]. This theory can be used to explain both expert competency and incompetency in software engineering tasks such as understanding code [69].

Phillips et al. [57] discuss how models containing tiny rule fragments can be quickly comprehended by doctors in emergency rooms making rapid decisions; or by soldiers on guard making snap decisions about whether to fire or not on a potential enemy; or by stockbrokers making instant decisions about buying or selling stock. That is, according to this psychological science theory $[9,14,22-$ $24,42,43,54,57]$, humans best understand a model:

- When they can "fit" it into their LTM; i.e., when that model comprises many small rule fragments;

- Further, to have an expert-level comprehension of some domain meaning having rules that can very quickly lead to decisions, without clogging up memory.

Psychological scientists have developed FFTs as one way to generate comprehensible models consisting of separate tiny rules [22, $42,57]$. A FFT is a decision tree with exactly two branches extending from each node, where either one or both branches is an exit branch leading to a leaf [42]. That is to say, in an FFT, every question posed by a node will trigger an immediate decision (so humans can read every leaf node as a separate rule).

For example, Table 2 (at left) is an FFT generated from the $\log 4 \mathrm{j}$ JAVA system of Table 3 . The goal of this tree is to classify a software module as "defective=true" or "defective=false". The four nodes in this FFT reference four static code attributes $c b o, r f c$, dam, amc (these metrics are defined in Table 1).

FFTs are a binary classification algorithm. To apply such classifiers to mulit-classes problems: (a) build one FFTs for each class for classX or not classX; (b) run all FFTs on the test example, then (c) then select conclusion with most support (number of rows).

An FFT of depth $d$ has a choice of two "exit policies" at each level: the existing branch can select for the negation of the target (denoted " 0 ") or the target (denoted " 1 "). The left-hand-side log $4 \mathrm{j}$ tree in Table 2 is hence an 01110 tree since:

- The first level exits to the negation of the target: hence, " 0 ".

- While the next tree levels exit first to target; hence, "111".

- And the final line of the model exits to the opposite of the penultimate line; hence, the final " 0 ".

To build one FFT tree, select a maximum depth $d$, then follow the steps described in Table 4

For trees of depth $d=4$, there are $2^{4}=16$ possible trees which we denoted $00001,00010,00101, \ldots, 11110$. Here, the first four digits

\footnotetext{
${ }^{2}$ Recently, Ma et al. [40] used evidence from neuroscience and functional MRIs to argue that STM capacity might be better measured using other factors than "number of items". But even they conceded that "the concept of a limited (STM) has considerable explanatory power for behavioral data".
} 
Table 1: The C-K OO metrics studied in Figure 1. Note that the last line. 'defect', denotes the dependent variable.

\begin{tabular}{|c|c|c|}
\hline Metric & Name & Description \\
\hline avg_cc & average McCabe & Average McCabe's cyclomatic complexity seen in class \\
\hline $\mathrm{ca}$ & afferent couplings & How many other classes use the specific class. \\
\hline cam & cohesion amongst classes & $\begin{array}{l}\text { Summation of number of different types of method parameters in every method divided by a multiplication of number } \\
\text { of different method parameter types in whole class and number of methods. }\end{array}$ \\
\hline cbm & coupling between methods & Total number of new/redefined methods to which all the inherited methods are coupled \\
\hline cbo & coupling between objects & Increased when the methods of one class access services of another. \\
\hline ce & efferent couplings & How many other classes is used by the specific class. \\
\hline dam & data access & Ratio of private (protected) attributes to total attributes \\
\hline lcom & lack of cohesion in methods & Number of pairs of methods that do not share a reference to an instance variable. \\
\hline locm3 & another lack of cohesion measure & $\begin{array}{l}\text { If } m, a \text { are the number of methods, attributes in a class number and } \mu(a) \text { is the number of methods accessing } \\
\text { an attribute, then } l \text { com } 3=\left(\left(\frac{1}{a} \sum_{j}^{a} \mu\left(a_{j}\right)\right)-m\right) /(1-m) \text {. }\end{array}$ \\
\hline loc & lines of code & Total lines of code in this file or package. \\
\hline max_cc & Maximum McCabe & maximum McCabe's cyclomatic complexity seen in class \\
\hline $\mathrm{mfa}$ & functional abstraction & Number of methods inherited by a class plus number of methods accessible by member methods of the class \\
\hline defect & defect & Boolean: where defects found in post-release bug-tracking systems. \\
\hline
\end{tabular}

Table 2: Three example FFTs.

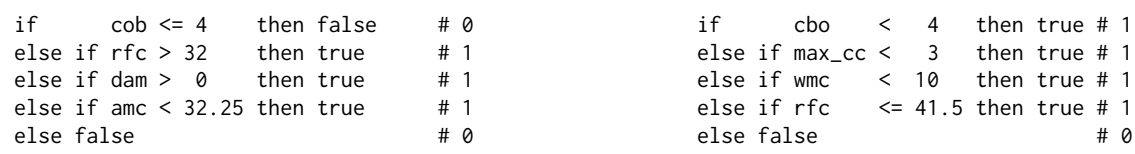

if $\quad$ dam $>0$ then false \# 0 else if noc $>0$ then false \# 0 else if wmc $>5$ then false \# 0 else if moa $>0$ then false \# 0 else true denote the 16 exit policies and the last digit denotes the last line of the model (which makes the opposite conclusion to the line above). For example:

- A "00001" tree does it all it can to avoid the target class. Only after clearing away all the non-defective examples it can at levels one, two, three, four does it make a final "true" conclusion. Table 2 (right) shows the $\log 4 \mathrm{j} 00001$ tree. Note that all the exits, except the last, are to "false".

- As to " 11110 " trees, these fixate on finding the target. Table 2 (center) shows the log $4 \mathrm{j} 11110$ tree. Note that all the exits, except the last, are to "true".

During FFT training, we generate all $2^{d}$ trees then, using the predicate score, select the best one (using the training data). This single best tree is then applied to the test data.

Table 3: Some open-source JAVA systems. Used for training and testing showing different details for each. All data available on-line at http://tiny.cc/seacraft.

\begin{tabular}{|l|l|l|l|l|l|}
\hline & Training & Testing & \\
\hline Data Set & Versions & Cases & Versions & Cases & \% Defective \\
\hline jedit & $3.2,4.0,4.1,4.2$ & 1257 & 4.3 & 492 & 2 \\
\hline ivy & $1.1,1.4$ & 352 & 2.0 & 352 & 11 \\
\hline camel & $1.0,1.2,1.4$ & 1819 & 1.6 & 965 & 19 \\
\hline synapse & $1.0,1.1$ & 379 & 1.2 & 256 & 34 \\
\hline velocity & $1.4,1.5$ & 410 & 1.6 & 229 & 34 \\
\hline lucene & $2.0,2.2$ & 442 & 2.4 & 340 & 59 \\
\hline poi & $1.5,2,2.5$ & 936 & 3.0 & 442 & 64 \\
\hline xerces & $1.0,1.2,1.3$ & 1055 & 1.4 & 588 & 74 \\
\hline log4j & $1.0,1.1$ & 244 & 1.2 & 205 & 92 \\
\hline xalan & $2.4,2.5,2.6$ & 2411 & 2.7 & 909 & 99 \\
\hline
\end{tabular}

Following the advice of [57], for all the experiments of this paper, we use a depth $d=4$. Note that FFTs of such small depths are very succinct (see above examples). Many other data mining algorithms used in software analytics are far less succinct and far less comprehensible (see Table 5).

The value of models such as FFTs comprising many small rules has been extensively studied:

- These models use very few attributes from the data. Hence they tend to be robust against overfitting, especially on small and noisy data, and have been found to predict data at levels comparable with regression. See for example [14, 42, 71].

\section{Table 4: Steps for building FFTs}

(1) First discretize all attributes; e.g., split numerics on median value.

(2) For each discretized range, find what rows it selects in the training data. Using those rows, score each range using some user-supplied score function e.g., recall, false alarm, or the $P_{o p t}$ defined in $\$ 3.4$.

(3) Divide the data on the best range.

(4) If the exit policy at this level is $(0,1)$, then exit to (false,true) using the range that scores highest assuming that the target class is (false,true), respectively.

(5) If the current level is at $d$, add one last exit node predicting the opposite to step 4 . Then terminate.

(6) Else, take the data selected by the non-exit range and go to step1 to build the next level of the tree. 
Table 5: Comprehension issues with models generated by data mining algorithms used in software analytics.

For very high dimensional data, there is some evidence that complex deep learning algorithms have advantages for software engineering applications $[25,68,72]$. However, since they do not readily support explainability, they have been criticizing as "data mining alchemy" [63].

Support vector machines and principle component methods achieve their results after synthesizing new dimensions which are totally unfamiliar to human users [50].

Other methods that are heavily based on mathematics can be hard to explain to most users. For example, in our experience, it is hard for (e.g.,) users to determine minimal changes to a project that mostly affect defect-proneness, just by browsing the internal frequency tables of a Naive Bayes classifier or the coefficients found via linear regression/logistic regression [50].

When decision tree learners are many pages long, they are hard to browse and understand [18].

Random forests are even harder to understand than decision trees since the problems of reading one tree are multiplied $N$ times, one for each member of the forest [38].

Instance-based methods do not compress their training data; instead they produce conclusions by finding older exemplars closest to the new example. Hence, for such instance-based methods, it is hard to generalize and make a conclusion about what kind of future projects might be (e.g.,) most defective-prone [4]

- Other work has shown that these rule-based models can perform comparably well to more complex models in a range of domains e.g., public health, medical risk management, performance science, etc. [28, 37, 59].

- Neth and Gigerenzer argue that such rule-bases are tools that work well under conditions of uncertainty [54].

- Brighton showed that rule-based models can perform better than complex nonlinear algorithms such as neural networks, exemplar models, and classification/regression trees [9].

\section{METHODS}

The use of models comprising many small rules has not been explored in the software analytics literature. This section describes the methods used by this paper to assess FFTs.

\subsection{Data}

3.1.1 Defect Data: To assess the FFTs, we perform our experiments using the publicly available SEACRAFT data [30], gathered by Jureczko et al. for object-oriented JAVA systems [30]. The "Jureczko" data records the number of known defects for each class using a post-release defect tracking system. The classes are described in terms of nearly two dozen metrics such as number of children (noc), lines of code (loc), etc (see Table 1). For details on the Jureczko data, see Table 3. The nature of collected data and its relevance to defect prediction is discussed in greater detail by Madeyski \& Jureczko [41].
Table 6: Metrics used in issue lifetimes data

\begin{tabular}{l|l|l} 
Commit & \multicolumn{1}{l}{ Comment } & \multicolumn{1}{l}{ Issue } \\
\hline nCommitsByActorsT & meanCommentSizeT & issueCleanedBodyLen \\
nCommitsByCreator & nComments & nIssuesByCreator \\
nCommitsByUniqueActorsT & & nIssuesByCreatorClosed \\
nCommitsInProject & & nIssuesCreatedInProject \\
nCommitsProjectT & & nIssuesCreatedInProjectClosed \\
& & nIssuesCreatedProjectClosedT \\
& & nIssuesCreatedProjectT \\
\hline Misc. & nActors, nLabels, nSubscribedByT \\
\hline
\end{tabular}

We selected these data sets since they have at least three consecutive releases (where release $i+1$ was built after release $i$ ). This is important for our experimental rig (see section 3.2).

3.1.2 Issue Lifetime Data: This paper will conclude that FFTs are remarkable effective. To check the external validity of that conclusion, we will apply FFT to another SE domain [60, 61]. Our Github issue lifetime data ${ }^{3}$ consists of 8 projects used to study issue lifetimes. In raw form, the data consisted of sets of JSON files for each repository, each file contained one type of data regarding the software repository (issues, commits, code contributors, changes to specific files as shown in Table 6). In order to extract data specific to issue lifetime, we did similar preprocessing and feature extraction on the raw datasets as suggested by [61].

\subsection{Experimental Rig}

For the defect prediction data, we use versions $i, j, k$ of the software systems in Table 3.

Using versions $i, j$, we track what attributes change by from version $i$ to $j$ (using the calculation shown in Figure 1). Then we build a model using all the attributes from version $j$ or just the top $25 \%$ most changed attributes. Note that this implements our definition of "operational", as discussed in our introduction.

After building a model, we use the latest version $k$ for testing while the older versions for training. In this way, we can assert that all our predictions are using past date to predict the future.

For the issue lifetime data, we do not have access to multiple versions of the data. Hence, for this data we cannot perform the operational test. Hence, for that data we conduct a $5^{*} 10$ crossvalidation experiment that ensures that the train and test sets are different. For that cross-val, we divide the data into ten bins, then for each bin $b_{i}$ we train on data $-b_{i}$ then test on bin $b_{i}$. To control for order effects (where the conclusions are altered by the order of the input examples) [2], this process is repeated five times, using different random orderings of the data.

\subsection{Data Mining Algorithms}

The results shown below compare FFTs to state of the art algorithms from software analytics. For a list of state-of-algorithms, we used the ICSE'15 paper from Ghotra et al. [21] which compared 32 classifiers for defect prediction. Their statistical analysis showed that the performance of these classifiers clustered into four groups shown in Table 7. For our work, we selected one classifier at random from each of their clusters: i.e., Simple Logistic (SL), Naive Bayes (NB),

\footnotetext{
${ }^{3}$ https://doi.org/10.5281/zenodo.197111
} 
Table 7: For the purposes of predicting software defects, Ghotra et al. [21] found that many learners have similar performance. Here are their four clusters of 32 data mining algorithms. For our work, we selected learners at random, one from each cluster (see the underlined entries).

\begin{tabular}{|c|c|c|c|c|}
\hline $\begin{array}{l}\text { Overall } \\
\text { Rank }\end{array}$ & $\begin{array}{l}\text { Classification } \\
\text { Technique }\end{array}$ & $\begin{array}{l}\text { Median } \\
\text { Rank }\end{array}$ & $\begin{array}{l}\text { Average } \\
\text { Rank }\end{array}$ & $\begin{array}{l}\text { Standard } \\
\text { Deviation }\end{array}$ \\
\hline 1 & $\begin{array}{l}\text { Rsub+J48, SL, Rsub+SL, } \\
\text { Bag+SL, LMT, RF+SL, } \\
\text { RF+J48, Bag+LMT, } \\
\text { Rsub+LMT, and RF+LMT }\end{array}$ & 1.7 & 1.63 & 0.33 \\
\hline 2 & $\begin{array}{l}\text { RBFs, Bag+J48, Ad+SL, } \\
\mathrm{KNN}, \mathrm{RF}+\mathrm{NB}, \mathrm{Ad}+\mathrm{LMT}, \\
\underline{\mathbf{N B}} \text {, Rsub+NB, and Bag+NB }\end{array}$ & 2.8 & 2.84 & 0.41 \\
\hline 3 & $\begin{array}{l}\text { Ripper, EM, J48, Ad+NB, } \\
\text { Bag+SMO, Ad+J48, } \\
\text { Ad+SMO, and K-means }\end{array}$ & 5.1 & 5.13 & 0.46 \\
\hline 4 & $\begin{array}{l}\text { RF+SMO, Ridor, SMO, } \\
\text { and Rsub+SMO }\end{array}$ & 6.5 & 6.45 & 0.25 \\
\hline
\end{tabular}

Expectation Maximization (EM), Sequential Minimal Optimization (SMO).

Simple Logistic and Naive Bayes falls into the 1st and 2nd rankings layers. They are both statistical techniques that are based on a probability based model [34]. These techniques are used to find patterns in datasets and build diverse predictive models [7]. Simple Logistic is a generalized linear regression model that uses a logit function. Naive Bayes is a probability-based technique that assumes that all of the predictors are independent of each other.

Clustering techniques like EM divide the training data into small groups such that the similarity within groups is more than across the groups [26]. EM is a clustering technique based on cluster performance Expectation Maximization [17] (EM) technique, which automatically splits a dataset into an (approximately) optimal number of clusters [8].

Support Vector Machines (SVMs) use a hyperplane to separate two classes (i.e., defective or not). In this paper, following the results of Ghotra et al., we use the Sequential Minimal Optimization (SMO) SVM technique. SMO analytically solves the large Quadratic Programming $(\mathrm{QP})$ optimization problem which occurs in SVM training by dividing the problem into a series of possible QP problems [75]

\subsection{Evaluation Measures}

Our rig assess learned models using an evaluation function called score. For FFTs, this function is called three times:

- Once to rank discretized ranges;

- Then once again to select the best FFT out of the $2^{d}$ trees generated during training.

- Then finally, score is used to score what happens when that best FFT is applied to the test data.

For all the other learners, score is applied on the test data. For this work, we use the two score measures: dis2heaven and $P_{\text {opt }}$.

Ideally, a perfect learner will have perfect recall (100\%) with no false alarms.

$$
\text { Recall }=\frac{\text { TruePositive }}{\text { TruePositive }+ \text { FalseNegative }}
$$

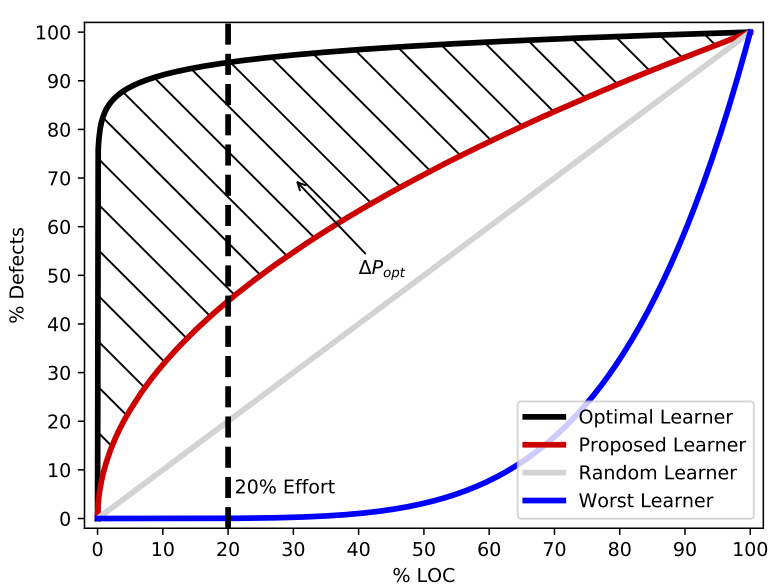

Figure 2: Effort-based cumulative lift chart [73].

$$
F A R=\frac{\text { FalsePositive }}{\text { FalsePositive }+ \text { TrueNegative }}
$$

We combine these two into a "distance to heaven" measure called dis2heaven that reports how far a learner falls away from the ideal point of Recall $=1$ and $F A R=0$ :

$$
\text { score }_{1}=\text { dis2heaven }=\sqrt{\frac{\left(1-\text { Recall }^{2}+F A R^{2}\right.}{2}}
$$

As to $P_{o p t}$, Ostrand et al. [56] report that their quality predictors can find $20 \%$ of the files contain on average $80 \%$ of all defects in the project. Although there is nothing magical about the number $20 \%$, it has been used as a cutoff value to set the efforts required for the defect inspection when evaluating the defect learners [31, 44, 53, 73]. That is, $P_{o p t}$ reports how many defects have been found after (a) the code is sorted by the learner from "most likely to be buggy" to "least likely"; then (b) humans inspect $20 \%$ of the code (measured in lines of code), where that code has, how many defects can be detected by the learner. This measure is widely used in defect prediction literature [31, 48, 49, 53, 73, 76].

$P_{o p t}$ is defined as $1-\Delta_{o p t}$, where $\Delta_{o p t}$ is the area between the effort cumulative lift charts of the optimal model and the prediction model (as shown in Figure2). In this chart, the $\mathrm{x}$-axis is the percentage of required effort to inspect the code and the $y$-axis is the percentage of defects found in the selected code. In the optimal model, all the changes are sorted by the actual defect density in descending order, while for the predicted model, all the changes are sorted by the actual predicted value in descending order. According to Kamei et al. and Xu et al. $[31,53,73] P_{\text {opt }}$ can be normalized as follows:

$$
\text { score }_{2}=P_{\text {opt }}(m)=1-\frac{S(\text { optimal })-S(m)}{S(\text { optimal })-S(\text { worst })}
$$

where $S$ (optimal), $S(\mathrm{~m})$ and $S$ (worst) represent the area of curve under the optimal model, predicted model, and worst model, respectively. This worst model is built by sorting all the changes according to the actual defect density in ascending order. 
Note that for our two score functions:

- For dis2heaven, the lower values are better.

- For $P_{o p t}$, the higher values are better.

\section{RESULTS}

\subsection{RQ1: Do FFTs models perform worse than the current state-of-the-art?}

Figure 3 compares the performance of FFT versus learners taken from Ghotra et al. In this figure, datasets are sorted left right based on the FFT performance scores. With very few exceptions:

- FFT's dis2heaven's results lower, hence better, than the other learners.

- FFT's $P_{\text {opt }}$ results are much higher, hence better, than the other learners.

Therefore our answer to RQ1 is:

For defect prediction, FFTs out-perform the state-of-art

When compared to state-of-the-art defect prediction algorithms surveyed by Ghotra et al., FFTs are more effective (where "effective" is measured in terms of a recall/false alarm metric or $\left.P_{\text {opt }}\right)$

\subsection{RQ2: Are FFTs more operational than the current state-of-the-art?}

Please recollect from before that a model is operational if its performance is not affected after avoiding attributes that are rarely changed by developers.

Figure 4 compares model performance when we learn from all $100 \%$ attributes or just the $25 \%$ most changed attributes. For this study, these $25 \%$ group (of most changed attributes) was computed separately for each data set. Note that:

- The top row of Figure 4 shows the dis2heaven results;

- The bottom row of Figure 4 shows the $P_{\text {opt }}$ results.

Figure 5 reports the deltas in performance scores seen between using $25 \%$ and $100 \%$ of the data. These deltas are computed such that larger values are better; i.e., for (dist2heaven, $\left.P_{\text {opt }}\right)$ we report $(25 \%$ $100 \%, 100 \%-25 \%$ ) since (fewer, more) values are better (respectively)

There are several key features for these results:

- The FFT's red dots for dis2heaven are below the rest; also, FFT's orange dots for $P_{o p t}$ are above the rest. This means that, regardless of whether we use all attributes or just the most changed attributes, the FFT results are nearly always better than the other methods.

- As seen in Figure 5, the deltas between using all data and just some of the data is smallest for FFTs and EM (the instance-based clustering algorithm). In $P_{o p t}$, those deltas are very small indeed (the FFT and EM results lie right on the y-axis for most of that plot).

- Also, see in Figure 5, the deltas on the other learners can be highly variable. While for the most part, using just the $25 \%$ most changed attributes improves performance, SMO, SL and NB all have large negative results for at least some of the data sets. In summary, the learners studied here fall into three groups:
Table 8: Frequency heatmap of best exit polices seen for FFT and defect prediction.

\begin{tabular}{c|r|r|r|r|r} 
Best FFF & & \multicolumn{2}{|c|}{$25 \%$} & \multicolumn{2}{|c}{$\mathbf{1 0 0 \%}$} \\
exit policy & & D2H & $P_{\text {opt }}$ & D2H & $P_{\text {opt }}$ \\
00001 & 0 & 0 & 0 & 0 & 0 \\
00010 & 0 & 0 & 0 & 0 & 0 \\
00101 & 0 & 0 & 0 & 0 & 0 \\
00110 & 0 & 0 & 0 & 0 & 0 \\
01001 & 0 & 0 & 0 & 0 & 0 \\
01010 & 0 & 0 & 0 & 0 & 0 \\
01101 & 1 & 0 & 0 & 0 & 1 \\
01110 & 0 & 0 & 0 & 0 & 0 \\
10001 & 14 & 6 & 0 & 7 & 1 \\
10010 & 8 & 4 & 2 & 2 & 0 \\
10101 & 3 & 0 & 1 & 1 & 1 \\
10110 & 5 & 0 & 3 & 0 & 2 \\
11001 & 0 & 0 & 0 & 0 & 0 \\
11010 & 3 & 0 & 1 & 0 & 2 \\
11101 & 2 & 0 & 0 & 0 & 2 \\
11110 & 4 & 0 & 3 & 0 & 1 \\
\hline Totals & 40 & 10 & 10 & 10 & 10
\end{tabular}

(1) Those that exhibited a wide performance variance after restricting the learning to just the frequently changed data (SL, $\mathrm{NB}, \mathrm{SMO}$ ), and those that are not (FFT, EM);

(2) Those with best performance across the two performance measures studied here (FFT), and the rest (SL, NB, EM, SMO);

(3) Those that generate tiny models (FFT), and the rest (SL, NB, EM, SMO).

Accordingly, FFT is the recommended learner since it both performs well and is unaffected by issues such as whether or not the data is restricted to just the most operational attributes. In summary:

When learning from less data, FFTs performance is stabler than some other learners.

When data is restricted to attributes that developers often change, then FFTs performance is only slightly changed while the performance of some other learners, can vary by alarmingly large amounts.

\subsection{RQ3: Why do FFTs work so well?}

To explain the success of FFTs, recall that during training, FFTs explores $2^{d}$ models, then selects the models whose exit policies achieves best performances (exit policies were introduced in Section 2.2). The exit policies selected by FFTs are like a trace of the reasoning jumping around the data. For example, a 11110 policy shows a model always jumping towards sections of the data containing most defects. Also, a 00001 policy show another model trying to jump away from defects until, in its last step, it does one final 

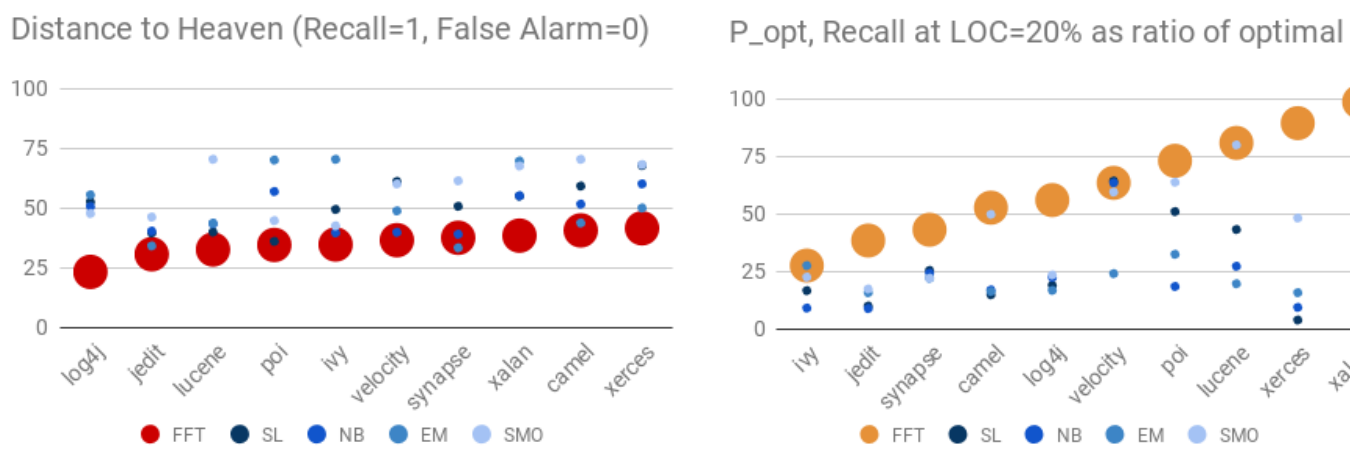

Figure 3: On the left, in the dis2Heaven results, less is better. On the right, in the $P_{o p t}$ results, more is better. On both sides, the FFTs results are better than those from state-of-the-art defect prediction algorithms (as defined by Ghotra et al. [21]).

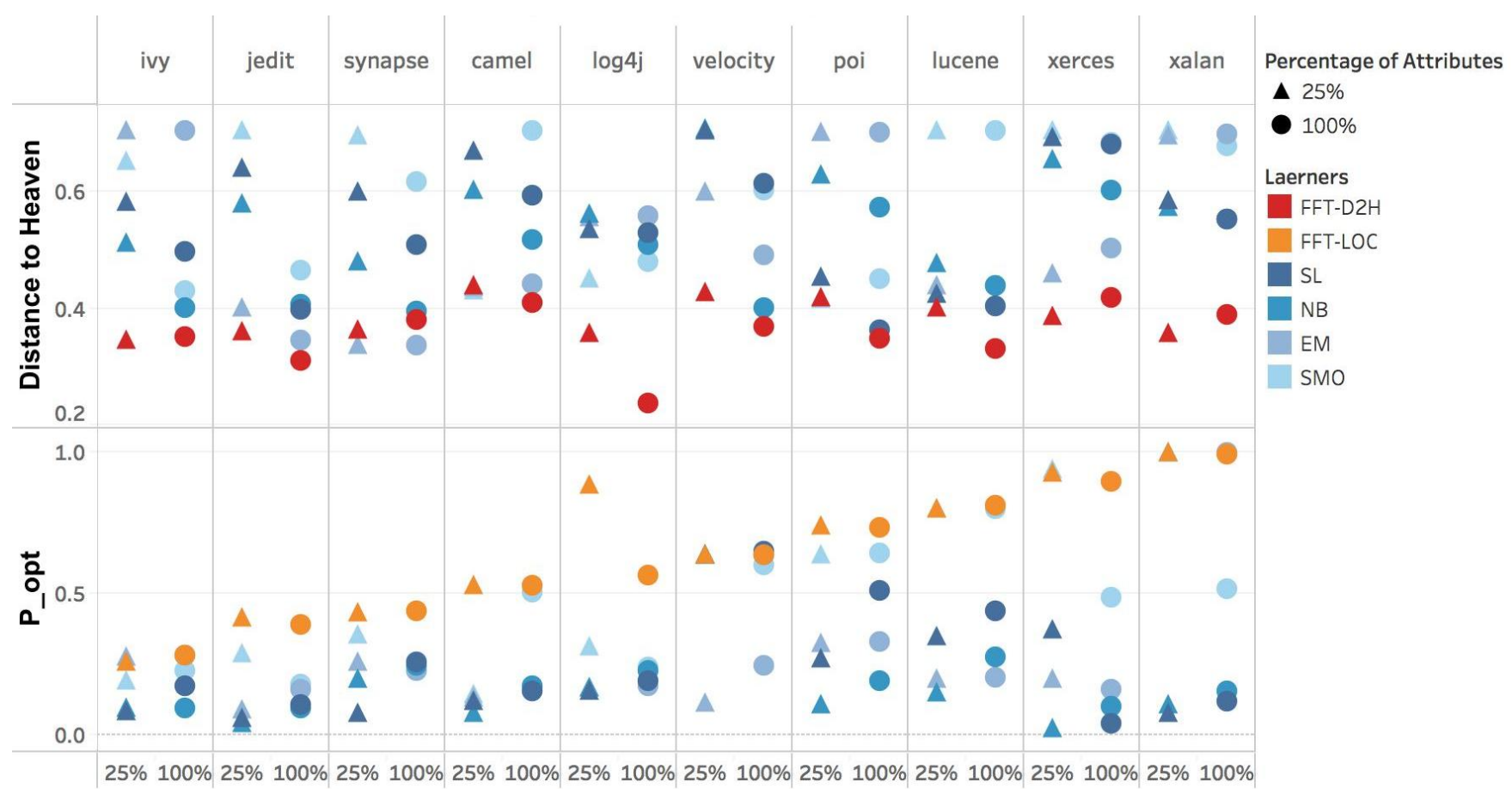

Figure 4: For each learner in Figure 3, this plot shows the difference between the results obtains using the top 25\% or all (100\%) of attributes. For (dist2heaven, $P_{o p t}$ ), values that are (lesser,greater) (respectively) are better. Note that all the $\bullet 100 \%$ results were also shown in Figure 3.

jump towards defects. Table 8 shows what exit policies were seen in the experiments of the last section:

- The 11110 policy was used sometimes.

- A more common policy is 10001 which shows a tree first jumping to some low hanging fruit (see the first " 1 "), then jumping away from defects three times (see the next " 000 ") before a final jump into defects (see the last " 1 ").

- That said, while 10001 was most common, many other exit policies appear in Table 8. For example, the $P_{o p t}$ policies are particularly diverse.

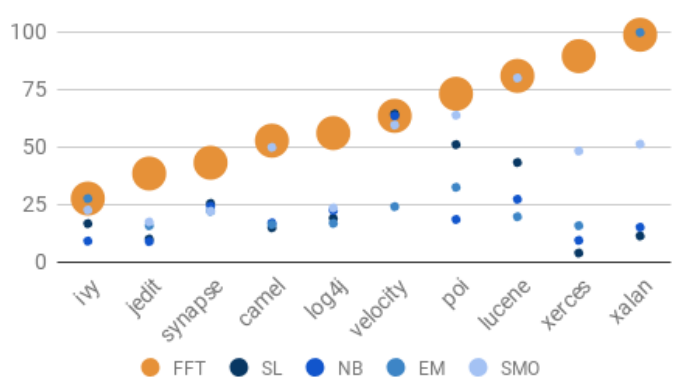




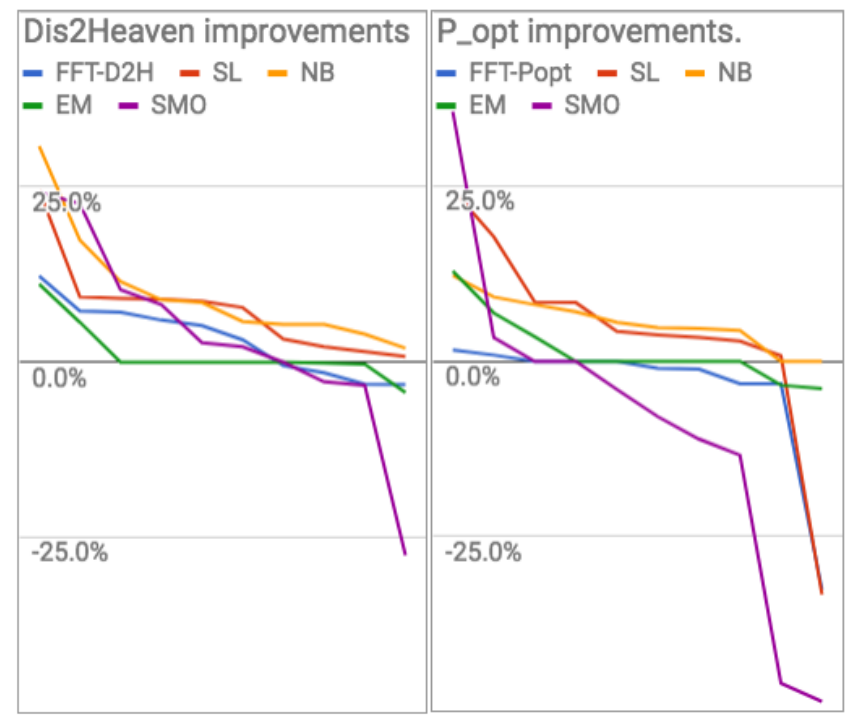

Figure 5: Deltas between results $25 \%$ and $100 \%$ of the data. Computed from Figure 4. Calculated such that larger values are better; i.e., for (dist2heaven, $\left.P_{\text {opt }}\right)$ we report $(25 \%-100 \%$, $100 \%-25 \%$ ) since (less, more) values are better (respectively). All values for each learner are sorted independently.

- Fit some general model across all the different parts of the data; e.g. like simple logistic regression.

In summary, in answer to the question "why do FFTs work so well", we reply:

\section{FFTs match the structure of SE data}

SE data divides into a few regions with very different properties and FFTs are good way to explore such data spaces.

\section{THREATS TO VALIDITY}

\subsection{Sampling Bias}

This paper shares the same sampling bias problem as every other data mining paper. Sampling bias threatens any classification experiment; what matters in one case may or may not hold in another case. For example, even though we use 10 open-source datasets in this study which come from several sources, they were all supplied by individuals.

As researchers, we can adopt two tactics to reduce the sampling bias problem. First we can document our tools and methods, then post an executable reproduction package for all the experiments (that package for this paper is available at url_blind_for_review).

Secondly, when new data becomes available, we can test our methods on the new data. For example, Table 9 shows results were FFTs and four different state-of-the-art learners, i.e. Decision Tree, Random Forest, Logistic Regression, K-Nearest Neighbors, were applied to the task of predicting issue close time (the other four learners were used since that was the technology recommended in a recent study in that domain $[60,61])$. Unlike the defect prediction
Table 9: Which learners performed better (in terms of median Dis2heaven) in $5^{*} 10$ cross-value experiments predicting for different classes of "how long to close an Github issue". Gray areas denote experiments where FFTs were outperformed by other learners. Note that, in (43/56=77\%) experiments, FFT performed better than the prior state-of-the-art in this area [60].

\begin{tabular}{|l|l|l|l|l|l|l|l|}
\hline \multirow{2}{*}{ Data(\# of instances) } & \multicolumn{7}{|c|}{ Days till closed } \\
\cline { 2 - 8 } & $>365$ & $<180$ & $<90$ & $<30$ & $<14$ & $<7$ & $<1$ \\
\hline cloudstack (1551) & FFT & FFT & FFT & FFT & FFT & DT & LR \\
\hline node (6207) & FFT & FFT & FFT & FFT & FFT & DT & LR \\
\hline deeplearning (1434) & FFT & FFT & FFT & FFT & FFT & FFT & RF \\
\hline cocoon (2045) & FFT & FFT & FFT & FFT & FFT & FFT & FFT \\
\hline ofbiz (6177) & FFT & FFT & FFT & FFT & FFT & FFT & FFT \\
\hline camel (5056) & RF/KNN & KNN & FFT/KNN/DT & FFT & FFT & FFT & FFT \\
\hline hadoop (12191) & KNN & DT & DT & FFT & FFT & FFT & FFT \\
\hline qpid (5475) & DT & DT/RF & DT & FFT & FFT & FFT & FFT \\
\hline
\end{tabular}

The goal here is to classify an issue according to how long it will take to close; i.e. less than 1 day, less than 7 days, and so on. Values collected via a $5 \times 10$ cross-validation procedure. Cells with a (white, gray) background means FFTs are statistically (better, worse) than (all, any) of the state-of-the-art learners (as determined by a Mann-Whitney test, $95 \%$ confidence) respectively. KNN, DT, RF and LR represents K-Nearest Neighbors, Decision Tree, Random Forest and Logistic Regression respectively.

data, we did not have multiple versions of the code so, for this domain, we used a $5^{*} 10$-way cross-validation analysis. White cells show where the FFT results were statistically different and better than all of the state-of-the-art learners' results. Note that, in most cases $(43 / 56=77 \%)$, FFTs performed better.

While this result does not prove that FFTs works well in all domains, it does show that there exists more than one domain where this is a useful approach.

\subsection{Learner Bias}

For building the defect predictors in this study, we elected to use Simple Logistic, Naive Bayes, Expectation Maximization, Support Vector Machine. We chose these learners because past studies shows that, for defect prediction tasks, these four learners represents four different levels of performance among a bunch of different learners $[3,21]$. Thus they are selected as the state-of-the-art learns to be compared with FFTs on the defect prediction data. While for Table 9), K-Nearest Neighbors, Decision Tree, Random Forest and Logistic Regression are used to compare against FFTs, because a recent work has summarized all the best learners that were applied on the issue lifetime data.

\subsection{Evaluation Bias}

This paper uses two performance measures, i.e., $P_{o p t}$ and dist 2 heaven as defined in Equation 4 and 3. Other quality measures often used in software engineering to quantify the effectiveness of prediction [29, 47, 51]. A comprehensive analysis using these measures may be performed with our replication package. Additionally, other measures can easily be added to extend this replication package.

\subsection{Order Bias}

For the performance evaluation part, the order that the data trained and predicted affects the results.

For the defect prediction datasets, we deliberately choose an ordering that mimics how our software projects releases versions 
so, for those experiments, we would say that bias was a required and needed.

For the issue close time results of Table 9, to mitigate this order bias, we ran our rig in a the 5-bin cross validation 10 times, randomly changing the order of the data each time.

\section{CONCLUSIONS}

This paper has shown that a data mining algorithm call Fast-andFrugal trees (FFTs) developed by psychological scientist is remarkably effective for creating actionable software analytics. Here "actionable" was defined as a combination of comprehensible and operational.

Measured in terms of comprehensibility, the FFT examples of Table 2 show that FFTs satisfy requirements raised by psychological scientists for "easily understandable at an expert level"; i.e., they comprise several short rules and those rules can be quickly applied (recall that each level of an FFT has an exit point which, if used, means humans can ignore the rest of the tree).

Despite their brevity, FFTs are remarkably effective:

- Measured in terms of $P_{o p t}$, FFTs are much better than other standard algorithms (see Figure 3).

- Measured in terms of distance to the "heaven" point of $100 \%$ recall and no false alarms, FFTs are either usually better than other standard algorithms used in software analytics (Random Forests, Naive Bayes, EM, Logistic Regression, and SVM). This result holds for at least two SE domains: defect prediction (see Figure 3) issue close time prediction (see Table 9).

As to being operational, we found that if learning is restricted to just the attributes changed most often, then the behavior of other learning algorithms can vary, wildly (see Figure 5). The behaviour of FFTs, on the other hand, remain remarkable stable across that treatment.

From the above, our conclusions is two-fold:

(1) There is much the software analytics community could learn from psychological science. FFTs, based on psychological science principles, out-perform a wide range of learners in widespread use.

(2) Proponents of complex methods should always baseline those methods against simpler alternatives. For example, FFTs could be used as a standard baseline learner against which other software analytics tools are compared.

\section{FUTURE WORK}

Numerous aspects of the above motivate deserve more attention.

\subsection{More Data}

This experiment with issue close time shows that FFTs are useful for more just defect prediction data. That said, for future work, it is important to test many other SE domains to learn when FFTs are useful. For example, at this time we are exploring text mining of StackOverflow data.

\subsection{More Learners}

The above experiments should be repeated, comparing FFTs against more learners. For example, at this time, we are comparing FFTs against deep learning for SE datasets. At this time, there is nothing as yet definitive to report about those results.

\subsection{More Algorithm Design}

These results may have implications beyond SE. Indeed, it might be insightful to another field-machine learning. For the reader familiar with machine learning literature, we note that FFTs are a decisionlist rule-covering model. FFTs restrict the (a) number of conditions per rule to only one comparison and (b) the total number of rules is set to a small number (often often just $d \in\{3,4,5\}$ ). Other decision list approaches such as PRISM [10], INDUCT [70],RIPPER [11] and RIPPLE-DOWN-RULES [20] produce far more complex models since they impose no such restriction. Perhaps the lesson of FFT is that PRISM,INDUCT,RIPPER, etc could be simplified with a few simple restrictions on the models they learn.

Also the success of FFT might be credited to its use on ensemble methods; i.e. train multiple times, then select the best. The comparison between FFTs and other ensemble methods like bagging and boosting [58] could be useful in future work.

\subsection{Applications to Delta Debugging}

There is a potential connection between the Figure 5 results and the delta debugging results of Zeller [74]. As shown above, we found that, sometimes focusing on the values that change most can sometimes, lead to better defect predictors (though, caveat empty or, sometimes it can actually make matters worse- see the large negative results in Figure 5). Note that this parallels Zeller's approach which he summarizes as "Initially, variable v1 was $\mathrm{x} 1$, thus variable $\mathrm{v} 2$ became $\mathrm{x} 2$, thus variable $\mathrm{v} 3$ became $\mathrm{x} 3 \ldots$ and thus the program failed". In future work, we will explore further applications of FFTs to delta debugging.

\section{REFERENCES}

[1] Behnoush Abdollahi and Olfa Nasraoui. 2016. Explainable restricted Boltzmann machines for collaborative filtering. arXiv preprint arXiv:1606.07129 (2016).

[2] Amritanshu Agrawal, Wei Fu, and Tim Menzies. 2018. What is Wrong with Topic Modeling?(and How to Fix it Using Search-based Software Engineering). Information and Software Technology (2018).

[3] Amritanshu Agrawal and Tim Menzies. 2018. Is "Better Data" Better than "Better Data Miners"? (Benefits of Tuning SMOTE for Defect Prediction). International Conference on Software Engineering (2018).

[4] David W Aha, Dennis Kibler, and Marc K Albert. 1991. Instance-based learning algorithms. Machine learning 6, 1 (1991), 37-66.

[5] A. Arcuri and L. Briand. 2011. A practical guide for using statistical tests to assess randomized algorithms in software engineering. In 2011 33rd International Conference on Software Engineering (ICSE). 1-10. DOI : http://dx.doi.org/10.1145/ 1985793.1985795

[6] Andrew Begel and Thomas Zimmermann. 2014. Analyze this! 145 questions for data scientists in software engineering. In Proceedings of the 36th International Conference on Software Engineering. ACM, 12-23.

[7] Alex Berson, Stephen Smith, and Kurt Thearling. 2004. An overview of data mining techniques. Building Data Mining Application for CRM (2004).

[8] Nicolas Bettenburg, Meiyappan Nagappan, and Ahmed E Hassan. 2012. Think locally, act globally: Improving defect and effort prediction models. In Proceedings of the 9th IEEE Working Conference on Mining Software Repositories. IEEE Press, 60-69.

[9] Henry Brighton. 2006. Robust Inference with Simple Cognitive Models.. In $A A A I$ spring symposium: Between a rock and a hard place: Cognitive science principles meet AI-hard problems. 17-22.

[10] Jadzia Cendrowska. 1987. PRISM: An algorithm for inducing modular rules. International Journal of Man-Machine Studies 27, 4 (1987), 349-370.

[11] William W. Cohen. 1995. Fast Effective Rule Induction. In ICML'95. 115-123.

[12] N. Cowan. 2001. The magical number 4 in short-term memory: a reconsideration of mental storage capacity. Behav Brain Sci 24, 1 (Feb 2001), 87-114. 
[13] Mark W Craven and Jude W Shavlik. 2014. Learning symbolic rules using artificial neural networks. In Proceedings of the Tenth International Conference on Machine Learning. 73-80.

[14] Jean Czerlinski, Gerd Gigerenzer, and Daniel G Goldstein. 1999. How good are simple heuristics? (1999).

[15] J. Czerwonka, R. Das, N. Nagappan, A. Tarvo, and A. Teterev. 2011. CRANE: Failure Prediction, Change Analysis and Test Prioritization in Practice - Experiences from Windows. In Software Testing, Verification and Validation (ICST), 2011 IEEE Fourth International Conference on. $357-366$.

[16] Hoa Khanh Dam, Truyen Tran, and Aditya Ghose. 2018. Explainable Software Analytics. arXiv preprint arXiv:1802.00603 (2018)

[17] Chris Fraley and Adrian E Raftery. 2007. Bayesian regularization for normal mixture estimation and model-based clustering. Journal of classification 24, 2 (2007), 155-181.

[18] Mark A Friedl and Carla E Brodley. 1997. Decision tree classification of land cover from remotely sensed data. Remote sensing of environment 61, 3 (1997), 399-409.

[19] Wei Fu and Tim Menzies. 2017. Easy over hard: a case study on deep learning. In Proceedings of the 2017 11th foint Meeting on Foundations of Software Engineering. ACM, 49-60.

[20] B. R. Gaines and P. Compton. 1995. Induction of Ripple-down Rules Applied to Modeling Large Databases. F. Intell. Inf. Syst. 5, 3 (Nov. 1995), 211-228. DOI: http://dx.doi.org/10.1007/BF00962234

[21] Baljinder Ghotra, Shane McIntosh, and Ahmed E Hassan. 2015. Revisiting the im pact of classification techniques on the performance of defect prediction models In Proceedings of the 37th International Conference on Software Engineering-Volume 1. IEEE Press, 789-800.

[22] Gerd Gigerenzer. 2008. Why heuristics work. Perspectives on psychological science 3,1 (2008), 20-29.

[23] Gerd Gigerenzer, Jean Czerlinski, and Laura Martignon. 1999. How good are fast and frugal heuristics. Decision science and technology: Reflections on the contributions of Ward Edwards (1999), 81-103.

[24] Gerd Gigerenzer and Wolfgang Gaissmaier. 2011. Heuristic decision making. Annual review of psychology 62 (2011), 451-482.

[25] Xiaodong Gu, Hongyu Zhang, Dongmei Zhang, and Sunghun Kim. 2016. Deep API learning. In Proceedings of the 2016 24th ACM SIGSOFT International Symposium on Foundations of Software Engineering. ACM, 631-642.

[26] Khaled Hammouda and Fakhreddine Karray. 2000. A comparative study of data clustering techniques. University of Waterloo, Ontario, Canada (2000).

[27] J. Hihn and T. Menzies. 2015. Data Mining Methods and Cost Estimation Models Why is it So Hard to Infuse New Ideas?. In 2015 30th IEEE/ACM International Conference on Automated Software Engineering Workshop (ASEW). 5-9. DOI : http://dx.doi.org/10.1109/ASEW.2015.27

[28] Mirjam A Jenny, Thorsten Pachur, S Lloyd Williams, Eni Becker, and Jürgen Margraf. 2013. Simple rules for detecting depression. Fournal of Applied Research in Memory and Cognition 2, 3 (2013), 149-157.

[29] Magne Jorgensen. 2004. Realism in assessment of effort estimation uncertainty: It matters how you ask. IEEE Transactions on Software Engineering 30, 4 (2004), 209-217.

[30] Marian Jureczko and Lech Madeyski. 2010. Towards identifying software project clusters with regard to defect prediction. In Proceedings of the 6th International Conference on Predictive Models in Software Engineering. ACM, 9.

[31] Yasutaka Kamei, Emad Shihab, Bram Adams, Ahmed E Hassan, Audris Mockus, Anand Sinha, and Naoyasu Ubayashi. 2013. A large-scale empirical study of just-in-time quality assurance. IEEE Transactions on Software Engineering 39, 6 (2013), 757-773.

[32] Miryung Kim, Thomas Zimmermann, Robert DeLine, and Andrew Begel. 2016 The Emerging Role of Data Scientists on Software Development Teams. In Proceed ings of the 38th International Conference on Software Engineering (ICSE '16). ACM New York, NY, USA, 96-107. DOI : http://dx.doi.org/10.1145/2884781.2884783

[33] E. Kocaguneli, T. Menzies, A. Bener, and J. Keung. 2012. Exploiting the Essential Assumptions of Analogy-Based Effort Estimation. IEEE Transactions on Software Engineering 28 (2012), 425-438. Issue 2. Available from http://menzies.us/pdf/ 11teak.pdf.

[34] Sotiris B Kotsiantis, I Zaharakis, and P Pintelas. 2007. Supervised machine learning: A review of classification techniques. (2007).

[35] Rahul Krishna and Tim Menzies. 2015. Actionable= Cluster+ Contrast?. In Automated Software Engineering Workshop (ASEW), 2015 30th IEEE/ACM International Conference on. IEEE, 14-17.

[36] Jill Larkin, John McDermott, Dorothea P. Simon, and Herbert A. Simon. 1980. Expert and Novice Performance in Solving Physics Problems. Science 208, 4450 (1980), 1335-1342. DOI : http://dx.doi.org/10.1126/science.208.4450.1335 arXiv:http://science.sciencemag.org/content/208/4450/1335.full.pdf

[37] K Laskey and Laura Martignon. 2014. Comparing fast and frugal trees and Bayesian networks for risk assessment. In Proceedings of the 9th International Conference on Teaching Statistics, Flagstaff, Arizona.

[38] Andy Liaw, Matthew Wiener, and others. 2002. Classification and regression by randomForest. $R$ news 2, 3 (2002), 18-22.
[39] Zachary C Lipton. 2016. The mythos of model interpretability. arXiv preprint arXiv:1606.03490 (2016).

[40] Wei Ji Ma, Masud Husain, and Paul M Bays. 2014. Changing concepts of working memory. Nature neuroscience 17, 3 (2014), 347-356.

[41] Lech Madeyski and Marian Jureczko. 2015. Which process metrics can significantly improve defect prediction models? An empirical study. Software Quality fournal 23, 3 (2015), 393-422.

[42] Laura Martignon, Konstantinos V Katsikopoulos, and Jan K Woike. 2008. Categorization with limited resources: A family of simple heuristics. Journal of Mathematical Psychology 52, 6 (2008), 352-361.

[43] Laura Martignon, Oliver Vitouch, Masanori Takezawa, and Malcolm R Forster. 2003. Naive and yet enlightened: From natural frequencies to fast and frugal decision trees. Thinking: Psychological perspectives on reasoning, judgment and decision making (2003), 189-211.

[44] Thilo Mende and Rainer Koschke. 2010. Effort-aware defect prediction models. In Software Maintenance and Reengineering (CSMR), 2010 14th European Conference on. IEEE, 107-116.

[45] Tim Menzies. 2014. OccamâĂŹs razor and simple software project management. In Software Project Management in a Changing World. Springer, 447-472.

[46] Tim Menzies, Alex Dekhtyar, Justin Distefano, and Jeremy Greenwald. 2007. Problems with Precision: A Response to "Comments on 'Data Mining Static Code Attributes to Learn Defect Predictors'". IEEE Transactions on Software Engineering 33, 9 (sep 2007), 637-640. DOI : http://dx.doi.org/10.1109/TSE.2007.70721

[47] Tim Menzies, Alex Dekhtyar, Justin Distefano, and Jeremy Greenwald. 2007. Problems with Precision: A Response to" comments on'data mining static code attributes to learn defect predictors'". IEEE Transactions on Software Engineering 33,9 (2007), 637-640.

[48] Tim Menzies, Jeremy Greenwald, and Art Frank. 2007. Data mining static code attributes to learn defect predictors. IEEE transactions on software engineering 33, 1 (2007), 2-13.

[49] Tim Menzies, Zach Milton, Burak Turhan, Bojan Cukic, Yue Jiang, and Ayşe Bener. 2010. Defect prediction from static code features: current results, limitations, new approaches. Automated Software Engineering 17, 4 (2010), 375-407.

[50] Tim Menzies, Osamu Mizuno, Yasunari Takagi, and Tohru Kikuno. 2009. Explanation vs Performance in Data Mining: A Case Study with Predicting Runaway Projects. Fournal of Software Engineering and Applications 2 (2009), 221-236.

[51] Tim Menzies, Dan Port, Zhihao Chen, and Jairus Hihn. 2005. Simple software cost analysis: safe or unsafe? In ACM SIGSOFT Software Engineering Notes, Vol. 30. ACM, 1-6.

[52] George A Miller. 1956. The magical number seven, plus or minus two: some limits on our capacity for processing information. Psychological review 63, 2 (1956), 81.

[53] Akito Monden, Takuma Hayashi, Shoji Shinoda, Kumiko Shirai, Junichi Yoshida, Mike Barker, and Kenichi Matsumoto. 2013. Assessing the cost effectiveness of fault prediction in acceptance testing. IEEE Transactions on Software Engineering 39,10 (2013), 1345-1357.

[54] Hansjörg Neth and Gerd Gigerenzer. 2015. Heuristics: Tools for an uncertain world. Emerging trends in the social and behavioral sciences: An interdisciplinary, searchable, and linkable resource (2015).

[55] Thomas J. Ostrand, Elaine J. Weyuker, and Robert M. Bell. 2004. Where the bugs are. In ISSTA '04: Proceedings of the 2004 ACM SIGSOFT international symposium on Software testing and analysis. ACM, New York, NY, USA, 86-96.

[56] Thomas J Ostrand, Elaine J Weyuker, and Robert M Bell. 2005. Predicting the location and number of faults in large software systems. IEEE Transactions on Software Engineering 31, 4 (2005), 340-355.

[57] Nathaniel D Phillips, Hansjoerg Neth, Jan K Woike, and Wolfgang Gaissmaier. 2017. FFTrees: A toolbox to create, visualize, and evaluate fast-and-frugal decision trees. Fudgment and Decision Making 12, 4 (2017), 344-368.

[58] J Ross Quinlan and others. 1996. Bagging, boosting, and C4. 5. In AAAI/IAAI, Vol. 1. $725-730$.

[59] Markus Raab and Gerd Gigerenzer. 2015. The power of simplicity: a fast-andfrugal heuristics approach to performance science. Frontiers in psychology 6 (2015).

[60] Tim Menzies Rahul Krishna. 2018. Bellwethers: A Baseline Method For Transfer Learning. arXiv preprint arXiv:1703.06218v4 (2018).

[61] Mitch Rees-Jones, Matthew Martin, and Tim Menzies. 2018. Better predictors for issue lifetime. Fournal of Software and Systems, submitted. arXiv preprint arXiv:1702.07735 (2018).

[62] Robert Sawyer. 2013. BIâĂŹs Impact on Analyses and Decision Making Depends on the Development of Less Complex Applications. In Principles and Applications of Business Intelligence Research. IGI Global, 83-95.

[63] AI Technology \& Industry Review Synced. 2017. LeCun vs Rahimi: Has Machine Learning Become Alchemy? (2017). https://medium.com/@Synced/ lecun-vs-rahimi-has-machine-learning-become-alchemy-21cb1557920d

[64] Shiang-Yen Tan and Taizan Chan. 2016. Defining and conceptualizing actionable insight: a conceptual framework for decision-centric analytics. arXiv preprint arXiv:1606.03510 (2016) 
[65] Christopher Theisen, Kim Herzig, Patrick Morrison, Brendan Murphy, and Laurie Williams. 2015. Approximating Attack Surfaces with Stack Traces. In ICSE'15.

[66] Burak Turhan, Ayşe Tosun, and Ayşe Bener. 2011. Empirical evaluation of mixed-project defect prediction models. In Software Engineering and Advanced Applications (SEAA), 2011 37th EUROMICRO Conference on. IEEE, 396-403.

[67] András Vargha and Harold D Delaney. 2000. A critique and improvement of the CL common language effect size statistics of McGraw and Wong. Fournal of Educational and Behavioral Statistics 25, 2 (2000), 101-132.

[68] Martin White, Christopher Vendome, Mario Linares-Vásquez, and Denys Poshy vanyk. 2015. Toward deep learning software repositories. In Mining Software Repositories (MSR), 2015 IEEE/ACM 12th Working Conference on. IEEE, 334-345.

[69] Susan Wiedenbeck, Vikki Fix, and Jean Scholtz. 1993. Characteristics of the mental representations of novice and expert programmers: an empirical study. International fournal of Man-Machine Studies 39, 5 (1993), 793-812.

[70] Ian H. Witten and Eibe Frank. 2002. Data Mining: Practical Machine Learning Tools and Techniques with Java Implementations. SIGMOD Rec. 31, 1 (March 2002), 76-77. DOI : http://dx.doi.org/10.1145/507338.507355

[71] Jan K Woike, Ulrich Hoffrage, and Laura Martignon. 2017. Integrating and testing natural frequencies, naïve Bayes, and fast-and-frugal trees. Decision 4, 4 (2017), 234.
[72] Xinli Yang, David Lo, Xin Xia, Yun Zhang, and Jianling Sun. 2015. Deep learning for just-in-time defect prediction. In Software Quality, Reliability and Security (QRS), 2015 IEEE International Conference on. IEEE, 17-26.

[73] Yibiao Yang, Yuming Zhou, Jinping Liu, Yangyang Zhao, Hongmin Lu, Lei Xu, Baowen Xu, and Hareton Leung. 2016. Effort-aware just-in-time defect prediction: simple unsupervised models could be better than supervised models. In Proceedings of the 2016 24th ACM SIGSOFT International Symposium on Foundations of Software Engineering. ACM, 157-168.

[74] Andreas Zeller. 2002. Isolating Cause-effect Chains from Computer Programs. In Proceedings of the 10th ACM SIGSOFT Symposium on Foundations of Software Engineering (SIGSOFT '02/FSE-10). ACM, New York, NY, USA, 1-10. DOI : http: //dx.doi.org/10.1145/587051.587053

[75] Zhi-Qiang Zeng, Hong-Bin Yu, Hua-Rong Xu, Yan-Qi Xie, and Ji Gao. 2008. Fas training support vector machines using parallel sequential minimal optimization. In Intelligent System and Knowledge Engineering, 2008. ISKE 2008. 3rd International Conference on, Vol. 1. IEEE, 997-1001.

[76] Thomas Zimmermann, Rahul Premraj, and Andreas Zeller. 2007. Predicting defects for eclipse. In Proceedings of the third international workshop on predictor models in software engineering. IEEE Computer Society, 9. 